\title{
Preparation and In Vitro Evaluation of Alginate Microparticles Containing Amphotericin B for the Treatment of Candida Infections
}

\author{
Merlis P. Alvarez-Berrios (iD, ${ }^{1}$ Lisa M. Aponte-Reyes, ${ }^{1}$ Lourdes Diaz-Figueroa, ${ }^{1}$ \\ Juan Vivero-Escoto, ${ }^{2}$ Alexis Johnston, ${ }^{2}$ and David Sanchez-Rodriguez ${ }^{1}$ \\ ${ }^{1}$ Department of Science and Technology, Inter American University of Puerto Rico, Ponce Campus, Ponce, PR, USA \\ ${ }^{2}$ Department of Chemistry, University of North Carolina at Charlotte, Charlotte, NC, USA
}

Correspondence should be addressed to Merlis P. Alvarez-Berrios; mpalvare@ponce.inter.edu

Received 25 January 2020; Revised 3 July 2020; Accepted 17 July 2020; Published 1 August 2020

Academic Editor: Chwee Teck Lim

Copyright (c) 2020 Merlis P. Alvarez-Berrios et al. This is an open access article distributed under the Creative Commons Attribution License, which permits unrestricted use, distribution, and reproduction in any medium, provided the original work is properly cited.

\begin{abstract}
Invasive candidiasis (IC) remains as a major cause of morbidity and mortality in critically ill patients. Amphotericin B (AmB) is one of the most effective antifungal agents commonly used to treat this infection. However, it induces severe side effects such as nephrotoxicity, cardiac alterations, nausea, fever, and liver damage. The utilization of drug delivery systems has been explored to overcome these limitations. Several AmB lipid formulations have been developed and are currently available in the market. Although they have the ability to reduce the main side effects of free AmB, their high cost, necessity of repeated intravenous injections for successful treatment, and incidence of pulmonary toxicity have limited their use. In the last decades, alginate has gained significant interest in drug delivery applications as a cost-effective strategy to improve the safety and therapeutic effect of toxic drugs. In this work, the clinically relevant drug AmB was encapsulated into alginate microparticles using the emulsification/ external gelation method. We hypothesize that this synthesis strategy may positively impact the antifungal efficacy of AmB-loaded MCPs toward Candida albicans cells while reducing the toxicity in human lung cells. To prove this hypothesis, the ability of the microplatform to disrupt the cellular membrane potential was tested and its antifungal effectiveness toward Candida albicans cells was evaluated using the cell counting and plate count methods. Moreover, the toxicity of the microplatform in human lung cells was evaluated using CellTiter $96^{\circledR}$ AQueous cell viability assay and qualitative diffusion analysis of acridine orange. Our results demonstrated that the platform developed in this work was able to induce antifungal toxicity against Candida albicans yeast cells at the same level of free AmB with minimal toxicity to lung cells, which is one of the main side effects induced by commercial drug delivery systems containing AmB. Overall, our data provides convincing evidence about the effectiveness of the alginate-based microplatform toward Candida albicans cells. In addition, this vehicle may not require several infusions for a successful treatment while reducing the pulmonary toxic effect induced by commercial lipid formulations.
\end{abstract}

\section{Introduction}

Invasive candidiasis (IC) is a serious infection caused by a yeast called Candida, with candidaemia being its most common clinical presentation [1-5]. One of the species responsible for this disease is Candida albicans which accounts for over $50 \%$ of all cases [6-8]. IC has been a major cause of morbidity and mortality in critically ill patients $[1,6,9]$. Particularly, patients that have HIV or diabetes mellitus or are undergoing a treatment that affects their immune system such as organ transplantation or chemotherapy are prone to obtain this type of infection [10]. IC affects more than 250,000 people every year and the mortality rate ranges from 40 to $70 \%[8,11-16]$. According to the Centers for Disease Control and Prevention (CDC), it is expected that approximately 25,000 cases of healthcare-associated invasive candidiasis occur each year in the United States $[1,17]$. 
One of the first-line therapies used to fight this infection is the polyene macrolide antibiotic amphotericin $\mathrm{B}(\mathrm{AmB})$ $[2,3,18,19]$. Its mechanism of action is based on the binding of the hydrophobic moiety of the molecule to the ergosterol of the fungal cell membrane, which disrupts the cellular membrane and causes the cytoplasmic content to leak out leading to cell death $[20,21]$. Unfortunately, this antimycotic drug induces severe side effects such as nephrotoxicity, cardiac alterations, nausea, fever, and liver damage $[3,22,23]$. The encapsulation of $\mathrm{AmB}$ into drug delivery systems has been explored as an alternative to improve its tolerability $[2,3,24]$. Currently, several lipid-based formulations such as the lipid complex Abelcet ${ }^{\circledR}$ and the liposomal product Ambisome ${ }^{\circledR}$ have been developed and are available in the market $[25,26]$. Although these lipid-based formulations reduce the main side effects of $\mathrm{AmB}$, their high cost, high-dose requirements, necessity of repeated intravenous injections for successful treatment, and incidence of pulmonary toxicity have limited their use [25-33]. Therefore, extensive research has been done to develop new lowcost approaches to improve the safety and therapeutic effect of this drug $[3,25,26,34,35]$. For example, AmB was encapsulated into PLGA nanoparticles. This vehicle showed lower toxicity when compared to free $\mathrm{AmB}$ and the in vitro antifungal effectivity was the same as that induced by the free drug [3]. On the other hand, the utilization of PLGA based fibrin microspheres as a delivery vehicle for $\mathrm{AmB}$ demonstrated that this platform was more effective in avoiding side effects when compared to the free drug and its antifungal efficacy was superior when compared to the free AmB [35]. In the last decades, alginate-based carriers have gained significant interest [34]. It is a naturally occurring polymer which is known to be biodegradable and biocompatible [36-38]. Several studies have developed alginate-based vehicles as carriers for various toxic drugs [34, 36, 39-41]. Nevertheless, only a few studies have fabricated alginatebased vehicles using different methods for the delivery of AmB [42, 43]. Sangeetha et al. formed a polyelectrolyte complex by mixing alginate and poly-L-lysine and encapsulated AmB into alginate/poly-L-lysine nanoparticles. The polyelectrolyte complex was formed to induce a slow and sustained release of the drug. The AmB-loaded alginate/ poly-L-lysine nanospheres were less toxic than the free drug. The authors suggested that the nanovehicle containing AmB was mainly accumulated in the lungs and liver, while the principal side effect of $\mathrm{AmB}$ was decreased due to the reduced uptake of the nanoparticles in the kidneys [43]. The in vitro antifungal toxicity was not performed. Another strategy to decrease the side effects of $A m B$ is the synthesis of alginate-AmB conjugates [42]. Ravichandran and Jayakrishnan obtained $\mathrm{AmB}$ conjugates by reacting $\mathrm{AmB}$ with sodium alginate via imine and amine linkages. The conjugates were encapsulated into gels discs of gelatin crosslinked with oxidized alginate. Although these conjugates were not toxic to human mammalian cells, the antifungal efficacy against Candida albicans yeast cells was less than that of the free drug. This may be attributed to the slow release of the drug. It was reported that the drug released from the gel discs was approximately $15-20 \%$ in 2 days [42].
Based on these results, alginate-based vehicles may confront similar clinical limitations as the commercial lipid formulations, requiring repeated intravenous injections for successful treatment and inducing pulmonary toxicity.

In this study, we encapsulated the clinically relevant drug AmB into alginate microparticles using the emulsification/ external gelation method. It has been reported that the production methods of alginate nano- or microparticles have a significant impact on tuning the (bio)pharmaceutical performance [44]. We hypothesize that this synthesis strategy may positively impact the antifungal efficacy of AmB-loaded MCPs toward Candida albicans cells while reducing the toxicity in human lung cells. To prove this hypothesis, the in vitro therapeutic efficacy of the microsystem toward Candida albicans was evaluated using the cell counting and plate count methods and through membrane depolarization studies using bis-(1,3-dibarbituric acid)-trimethine oxonol [ $\left.\mathrm{DiBAC}_{4}(3)\right]$. Moreover, the toxicity of the microplatform in human lung cells was evaluated using CellTiter $96^{\circledR}$ AQueous cell viability assay and qualitative diffusion analysis of acridine orange. To our knowledge, no study has been carried out to study the in vitro effectiveness and toxicity of alginate microparticles containing $\mathrm{AmB}$ using emulsification/external gelation method as described in this work.

Our results demonstrated that AmB-loaded MCPs were able to disrupt the cellular membrane potential of Candida albicans yeast cells and the in vitro antifungal effectivity was the same as that induced by the free drug. Moreover, the microvehicle showed minimal lung cell toxicity and cell membrane damage. Overall, this data provides convincing evidence of the effectiveness of the platform developed in this work toward Candida albicans cells, which in turn may reduce the side effect of current vehicles associated with pulmonary toxicity.

\section{Materials and Methods}

2.1. Materials. Low viscosity alginic acid sodium salt, calcium chloride, amphotericin $\mathrm{B}$, isopropanol, span 80 , bis(1,3-dibutylbarbituric acid) trimethine oxonol (DiBAC $4(3)$ ), and paraffin oil were obtained from Sigma-Aldrich (St. Louis, MO, USA) and were used as received without further purification. Roswell Park Memorial Institute (RPMI 1640), penicillin-streptomycin, phosphate buffer saline (PBS, 1X), and trypsin were purchased from Corning. Fetal bovine serum (FBS) was purchased from Atlanta Biologicals. CellTiter $96{ }^{\circledR}$ AQueous Assay was obtained from Promega (Madison, WI, USA). Acridine orange solution was purchased from Invitrogen.

A Multiskan FC plate reader by Fisher Scientific was used for the cell viability analysis. An Olympus Fluoview FV 1000 confocal fluorescence microscope system was used for the qualitative diffusion analysis experiments.

2.2. Synthesis and Characterization of AmB-Loaded MCPs. AmB-loaded MCPs were fabricated using an emulsification method previously reported in the literature with some 
modifications [45]. Briefly, $10 \mathrm{mg}$ of $\mathrm{AmB}$ was dissolved in $10 \mathrm{~mL}$ of an aqueous solution of alginate $(5 \% \mathrm{wt} / \mathrm{vol})$. Then, the alginate solution containing $\mathrm{AmB}$ was dispersed into a mixture of paraffin oil and span $80(40 \mathrm{~mL}$ of paraffin oil and $2 \mathrm{~mL}$ of spam 80) at $1200 \mathrm{rpm}$ for 30 minutes. Afterward, $20 \mathrm{~mL}$ of a $10 \% \mathrm{CaCl}_{2}$ solution was added and stirred at $800 \mathrm{rpm}$ for an additional 2 hours. After this period of time, $20 \mathrm{~mL}$ of isopropanol was added and the mixture was allowed to react for 10 minutes. The final product was separated by centrifugation, washed three times with isopropanol, let dry at room temperature, and stored at $-20^{\circ} \mathrm{C}$. AmB-loaded MCPs were characterized using different techniques: the hydrodynamic diameter and $\zeta$-potential were determined using a particle size analyzer (Nanoplus HD, Particulate Systems, GA, USA), particle size and morphology were estimated using an optical microscope (Nikon, Nikon Instruments, NY, USA) and field-emission scanning electron microscopy (SEM; Raith150, Raith, Dortmund, Germany), and the FTIR spectrum and the absorption spectra were determined using an IR spectrophotometer (Spectrum Two, Perkin Elmer, CT, USA) and UV-Vis spectrophotometer (Evolution 260 Bio, Thermo Fisher Scientific, MA, USA), respectively.

2.3. AmB Content and Encapsulation Efficiency. The amount of $\mathrm{AmB}$ encapsulated into alginate microparticles was determined by dissolving $10 \mathrm{mg}$ of AmB-loaded MCPs in $3 \mathrm{~mL}$ of PBS ( $\mathrm{pH} 7.4$ ) under stirring conditions for 24 hours. After this period of time, the sample was centrifuged and the supernatant was analyzed using a spectrophotometer at a wavelength of $345 \mathrm{~nm}[43,46]$. The concentration of AmB was determined using a calibration curve from $4 \mu \mathrm{g} / \mathrm{mL}$ to $65 \mu \mathrm{g} / \mathrm{mL}$ of AmB. $345 \mathrm{~nm}$ is the wavelength at which AmB in aqueous solution shows its maximum absorbance [47]. $\mathrm{AmB}$ content was established as the amount of $\mathrm{AmB}(\mu \mathrm{g})$ per mg of microparticles. The percentage of encapsulation was calculated by dividing the amount of $\mathrm{AmB}$ encapsulated by the initial amount of $A \mathrm{mB}$ added to the alginate solution. The experiment was carried out in triplicate.

2.4. Depolarization Studies. It is known that damage induced to cellular membranes may cause the dissipation of the membrane potential $[48,49]$. Depolarization studies were performed to evaluate the ability of AmB-loaded MCPs to disrupt the cellular membrane of Candida albicans cells. For this experiment, the membrane potential-sensitive probe bis(1,3-dibutylbarbituric acid) trimethine oxonol $\left(\mathrm{DiBAC}_{4}(3)\right)$ was used. $\mathrm{DiBAC}_{4}(3)$ is an anionic dye which only fluoresces in the microenvironment of the membrane. Normal cells have a negative internal charge and exclude the dye. When cells are exposed to $\mathrm{AmB}$, the drug disrupts the membrane allowing the dye to enter and fluoresce [48, 49]. An increase in fluorescence after adding AmB indicates its ability to disrupt the cellular membrane. To carry out the assay, Candida albicans cells purchased from ATCC (ATCC 10231) were cultured in yeast extract peptone dextrose (YPD) agar at $37^{\circ} \mathrm{C}$ for 24 hours. Then, approximately four colonies were resuspended in sterile saline water, the concentration was determined using a hemocytometer, and $2 \times 10^{6}$ cells were resuspended in $2.5 \mathrm{~mL}$ of sterile saline water. The fluorescence of the cell suspension was followed up for 1-2 minutes to obtain a baseline. Afterward, $\operatorname{DiBAC}_{4}(3)$ was added at a final concentration of $22.5 \mu \mathrm{g} / \mathrm{ml}$ and the fluorescence was monitored for 90 seconds until a stable signal was obtained. Then, free AmB or AmB-loaded MCPs were added and changes in the membrane potential were monitored. The fluorescence for this experiment was determined using a spectrofluorometer at an excitation wavelength of $490 \mathrm{~nm}$. The measurements were repeated two times.

2.5. In Vitro Release Assay. To verify that $\mathrm{AmB}$ is able to be released from the microparticles, $10 \mathrm{mg}$ of AmB-loaded MCPs was suspended in $2 \mathrm{~mL}$ of PBS under stirring at $200 \mathrm{rpm}$. At specific time intervals, $100 \mu \mathrm{L}$ of the sample was withdrawn and centrifuged and the supernatant was collected and analyzed spectrophotometrically at $345 \mathrm{~nm}$. The experiment was carried out in triplicate.

2.6. Antifungal Activity of AmB-Loaded MCPs against Candida albicans Cells. The effectiveness of the platform developed in this work against Candida albicans cells was evaluated using the cell counting method and plate count method. To compare the effect of free AmB and AmB-loaded MCPs at the same conditions, the amount of AmB-loaded MCPs added corresponded to an equivalent amount of free AmB.

Candida albicans cells were obtained from American Type Culture Collection (ATCC 10231) and grown on YPD agar plates (1\% yeast extract, $2 \%$ peptone, $2 \%$ dextrose, and $2 \%$ bacteriological agar) at $35^{\circ} \mathrm{C}$ for 24 hours. Then, approximately two colonies were transferred to a tube containing $10 \mathrm{~mL}$ of YPD medium and homogenized. The concentration of the cell suspension was determined using a hemocytometer and $5 \times 10^{3}$ cells were added to tubes containing different concentrations of $\mathrm{AmB}(0.5 \mu \mathrm{g} / \mathrm{mL}$ or $4 \mu \mathrm{g} /$ $\mathrm{mL}$ ). The concentrations of $\mathrm{AmB}$ used for this study were chosen in accordance with clinical concentrations reported in the literature $[50,51]$. The sterile test tubes were incubated for 48 hours at $35^{\circ} \mathrm{C}$ and $250 \mathrm{rpm}$. After the treatment, the viability ratio was determined by cell counting using a hemocytometer. For the plate count method, $2.5 \times 10^{2}$ cells were spread on YPD agar plates and incubated at $35^{\circ} \mathrm{C}$ for 48 hours. The number of viable cells was counted and expressed in $\mathrm{CFU} / \mathrm{mL}$. The cytotoxic effect of blank microparticles (MCPs) was evaluated at the concentration of microparticles corresponding to $0.5 \mu \mathrm{g} / \mathrm{mL}$ and $4 \mu \mathrm{g} / \mathrm{mL}$ of $\mathrm{AmB}$. The experiment was performed in duplicate.

2.7. Cell Culture. Because the majority of the studies related to the fabrication of polymeric vehicles have focused on evaluating their in vitro toxicity in human kidney cells, demonstrating their ability to significantly decrease the main side effects of AmB [25, 26, 42, 52, 53]; this study will only focus on lung cell toxicity which is one of the main side effects related to commercial formulations containing AmB. 
Immortalized or cancer cells lines are widely used for in vitro toxicology studies [54]. H-460, a human large cell lung cancer, was purchased from American Type Culture Collection (ATCC). H-460 cells were cultured in RPMI 1640 medium supplemented with $10 \%$ FBS and $1 \%$ pen-step at $37^{\circ} \mathrm{C}$ with $5 \% \mathrm{CO}_{2}$ atmosphere. The culture media were changed every other day. All cell cultures were maintained in $25 \mathrm{~cm}^{2}$ or $75 \mathrm{~cm}^{2}$ cell culture flasks and the cells were passaged at $70-80 \%$ confluency every $2-4$ days.

2.8. In Vitro Cytotoxicity. For this study, H-460 cells were seeded in a 96-well plate at a density of 1,000 cells per well in $100 \mu \mathrm{L}$ of complete media. The cells were incubated at $37^{\circ} \mathrm{C}$ with $5 \% \mathrm{CO}_{2}$ atmosphere for $48 \mathrm{~h}$. After removing the cell culture medium, AmB at different concentrations (10, 30, 50, and $100 \mu \mathrm{g} / \mathrm{mL}$ ) was added to the cells. To compare the effect of free AmB and AmB-loaded MCPs at the same conditions, the amount of AmB-loaded MCPs added corresponded to an equivalent amount of free AmB. Blank MCPs were used as a control at the concentrations used for AmB-loaded MCPs. After $48 \mathrm{~h}$ of incubation in the presence of these materials, the culture media were removed and fresh media $(100 \mu \mathrm{L})$ together with $20 \mu \mathrm{L}$ of CellTiter $96{ }^{\circledR}$ Aqueous solution were added. The cells were incubated for $2-3 \mathrm{~h}$ at $37^{\circ} \mathrm{C}$ with $5 \%$ $\mathrm{CO}_{2}$ atmosphere. Cell viability (\%) was calculated as follows: viability $=\left(A_{\text {sample }} / A_{\text {control }}\right) \times 100 \%$, where $A_{\text {sample }}$ and $A_{\text {control }}$ denote absorbance values of the sample and control wells measured at $490 \mathrm{~nm}$, respectively. The results are reported as the average \pm SE of four experiments.

2.9. Confocal Microscopy. H-460 cells were seeded at a concentration of $5 \times 10^{4}$ cells per well on a glass cover slip in a 6-well plate with $2 \mathrm{~mL}$ of complete media and incubated for $24 \mathrm{~h}$ at $37^{\circ} \mathrm{C}$ and $5 \% \mathrm{CO}_{2}$ atmosphere. The cells were exposed to free $\mathrm{AmB}$ or AmB-loaded MCPs at an equivalent concentration of $50 \mu \mathrm{g} / \mathrm{mL}$ of $\mathrm{AmB}$ and incubated for $24 \mathrm{~h}$. Afterward, the cells were washed three times with PBS and incubated with acridine orange (AO) solution $(1 \mu \mathrm{g} / \mathrm{mL}$, $2 \mathrm{~mL}$ ) for $5 \mathrm{~min}$ at room temperature. After incubation with $\mathrm{AO}$, the cells were washed twice with PBS and the glass cover-slips were mounted on a microscope slide using a spacer. Cells without any treatment were used as controls. The cells were imaged using an Olympus FluoView FV 1000 confocal microscope.

2.10. Statistical Analysis. Statistical analyses were conducted using Student's $t$-test (two-tailed distribution, two samples with unequal variances). Differences were considered significant at $P<0.05$.

\section{Results and Discussion}

3.1. Characterization of $A m B$-Loaded MCPs. In this work, an alginate-based microsystem for the delivery of the clinically relevant antifungal antibiotic $\mathrm{AmB}$ was fabricated using an emulsification method followed by cross-linking with calcium chloride [45]. The resulting AmB-loaded MCPs had a diameter of $1.3 \pm 0.3 \mu \mathrm{m}$ according to SEM and optical microscope (Figure 1). The hydrodynamic diameter was $1.6 \pm 0.4 \mu \mathrm{m}$ as determined by DLS (Figure 2(a)). The $\zeta$-potential of the microsystem was $-40.8 \pm 2.2 \mathrm{mV}$. These results demonstrated that the synthesized AmB-loaded MCPs were not aggregated in solution as shown by the similarities in size obtained by DLS and SEM images (no statistically significant difference was observed between sizes obtained by SEM and DLS). This may be attributed to the large negative value of $\zeta$-potential that prevents aggregation due to electrostatic repulsion of individual microparticles $[55,56]$. Blank microparticles (MCPs) showed a diameter of $1.6 \pm 0.3 \mu \mathrm{m}$ according to SEM and optical microscope. This indicates that the incorporation of $\mathrm{AmB}$ did not induce an increase in the diameter of the microplatform.

The FT-IR spectrum of AmB-loaded MCPs showed the characteristic absorption bands of AmB. Figure 2(c) shows the stretching vibrations at $2923 \mathrm{~cm}^{-1}$ and $3349 \mathrm{~cm}^{-1}$ corresponding to $\mathrm{CH}_{2}$ and $\mathrm{CH}_{3}$ groups and $\mathrm{OH}$, respectively $[57,58]$. On the other hand, the absorption spectra of AmBloaded MCPs showed the characteristic absorption peaks of $\mathrm{AmB}$ confirming the successful encapsulation of AmB into the alginate microparticles while remaining stable and intact (Figure 2(b)). The absorption spectra provide additional information about the aggregation state of AmB [42, 47]. Aggregates are responsible for inducing toxicity in mammalian cells and the ratio of absorbance of the first and fourth peak in the absorption spectra provides information regarding the extent of aggregation of $\mathrm{AmB}$ (a value less than 1 is characteristic of the monomeric form, while a value higher than 2 corresponds to the aggregated form) [47]. The ratio of the first and fourth peak for the AmB-loaded MCPs was 1.3 indicating that this vehicle may induce low toxicity.

The amount of AmB loaded on the microparticles was $6.11 \pm 0.06 \mu \mathrm{g}$ of $\mathrm{AmB} / \mathrm{mg}$ of microparticles and the percentage of encapsulation was $15.8 \%$ as determined by UVVis spectroscopy.

3.2. In Vitro Release Profile. An attractive aspect of polymeric-based vehicles is their ability to promote sustained release of drugs at concentrations capable of inducing a therapeutic effect. To evaluate the release of AmB from the alginate-based vehicle synthesized in this work, an in vitro drug release assay was performed. The results demonstrated that $\mathrm{AmB}$ was released from the alginate-based vehicle as shown in Figure 3. A burst of AmB released from the alginate vehicle was observed at the initial stage, followed by a slower release phase. During the first 60 minutes, 15\% of the drug was released, and at 120 minutes $21 \%$ of the drug was released. This release behavior is similar to those reported in the literature [36]. An interesting aspect observed in the release profile is that the amount of drug released from our platform is higher than other alginate-based vehicles reported in the literature that have shown lower in vitro antifungal toxicity when compared to the free drug. For example, Ravichandran and Jayakrishnan encapsulated alginate-AmB conjugates into gels discs of gelatin cross-linked with oxidized alginate. The authors reported that the release 


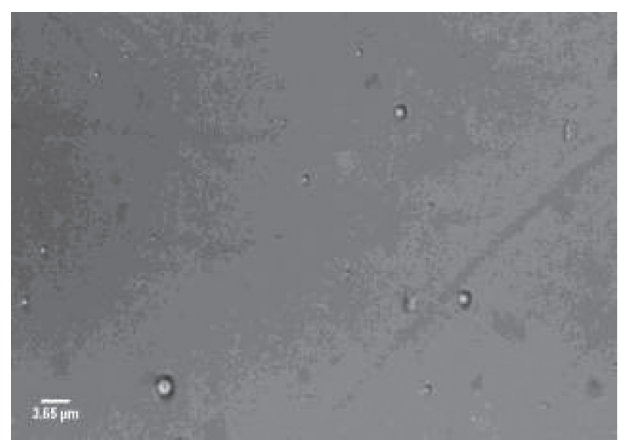

(a)

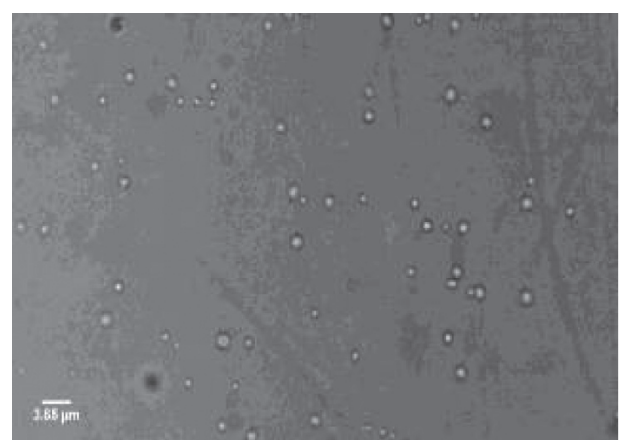

(c)

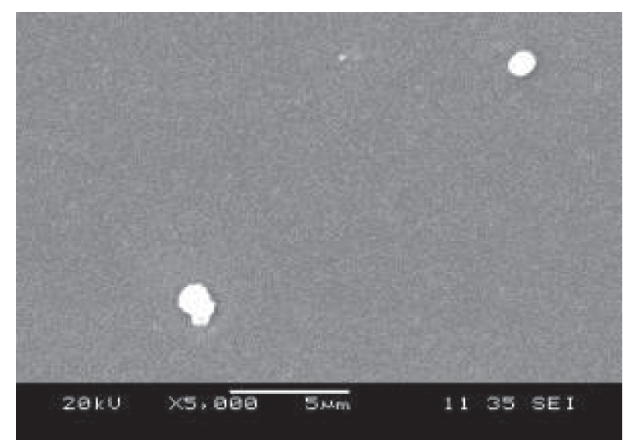

(b)

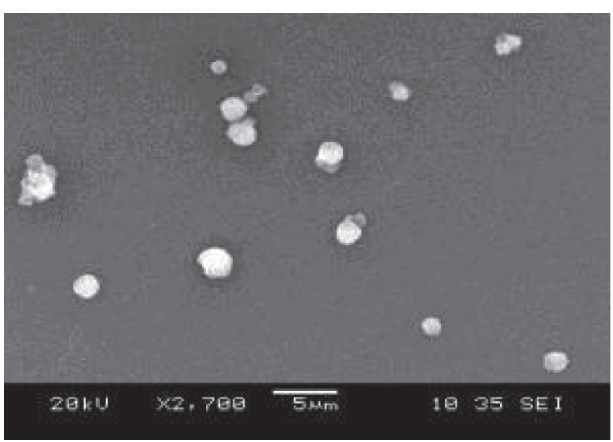

(d)

FIGURE 1: Particle size and morphology of alginate-based microparticles. Optical microscope (a) and SEM (b) images of AmB-loaded MCPs. Optical microscope (c) and SEM (d) images of blank microparticles (MCPs).

from the gel discs was approximately $15-20 \%$ in 2 days and the antifungal efficacy against Candida albicans yeast was less than that of the free drug [42]. On the other hand, De Castro et al. fabricated alginate nanoparticles containing miltefosine. The results demonstrated that the in vitro effectiveness of the platform toward Candida spp. was lower than the free drug. The authors established that these results were correlated to the slow release of the free drug from alginate nanoparticles which was only $7.55 \%$ in $24 \mathrm{~h}$ [36].

Because one of the main concerns of current AmB formulations is the requirement for repeated intravenous injections for successful treatment resulting in high costs, it is important to develop formulations that promote a sustained release of drugs at concentrations that will induce a therapeutic effect similar to or higher than the free drug. Based on the release profile, we anticipate that our platform will induce an in vitro antifungal activity toward Candida albicans.

3.3. Evaluation of Activity of AmB-Loaded MCPs. The mechanism of action of $\mathrm{AmB}$ is based on the binding of the hydrophobic moiety of the molecule to the ergosterol found in the fungal cell membrane, leading to cell membrane damage and subsequent death of the microorganism [20]. Membrane depolarization studies were performed to evaluate the ability of the $\mathrm{AmB}$ encapsulated into the alginate microparticles to disrupt the cellular membrane of Candida albicans cells. To carry out this experiment, the anionic fluorescent probe $\mathrm{DiBAC}_{4}(3)$ which only fluoresces in the microenvironment of the membrane was used. Normal cells exclude the dye because they have a negative internal charge. However, exposure to AmB induces membrane depolarization, which allows the dye to enter, bind to lipid-rich intracellular components, and fluoresce $[48,49,59]$. Thus, an increase in fluorescence after exposure to $\mathrm{AmB}$ indicates its ability to disrupt the cellular membrane. The results demonstrated that the microvehicle developed was able to disrupt the cellular membrane potential at the same level as free AmB (Figure 4). The first 120 seconds of Figure 4 shows a baseline corresponding to cells suspended in saline water. After addition of the fluorescent probe, an increase in intensity was observed due to the presence of the fluorescent compound; the fluorescence was measured until a stable signal intensity was achieved. After adding AmB or AmBloaded MCPs, a significant increase in fluorescence was observed, suggesting the induction of membrane depolarization. This indicated that the activity of $A m B$ was not compromised during the encapsulation process. In addition, the AmB released from the alginate-based vehicle may induce a therapeutic effect toward Candida albicans yeast at the same level as the free drug which in turn may result in avoiding the requirement for repeated intravenous injections for successful treatment.

3.4. Antifungal Efficacy of AmB-Loaded MCPs toward Candida albicans Yeasts. It has been reported that Candida albicans is responsible for over $50 \%$ of all cases of invasive candidiasis [6,7]. Therefore, Candida albicans cells were 


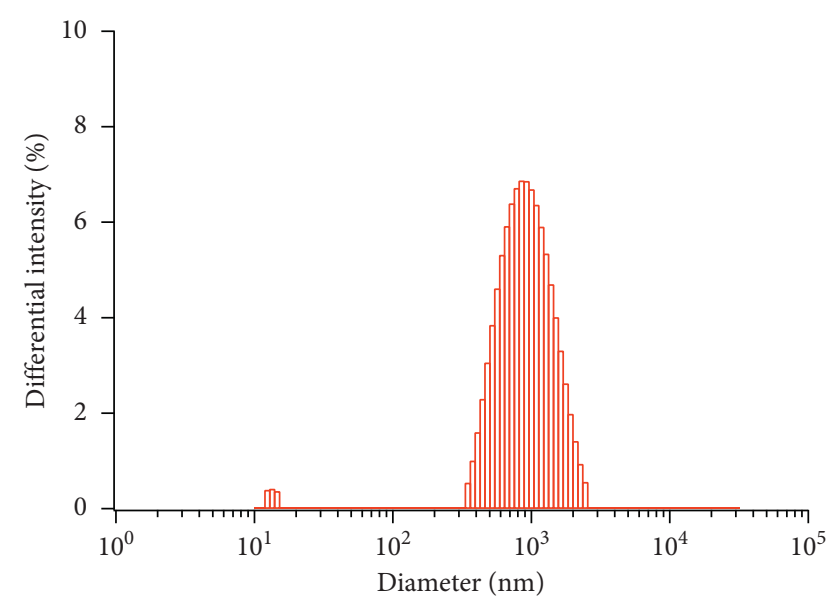

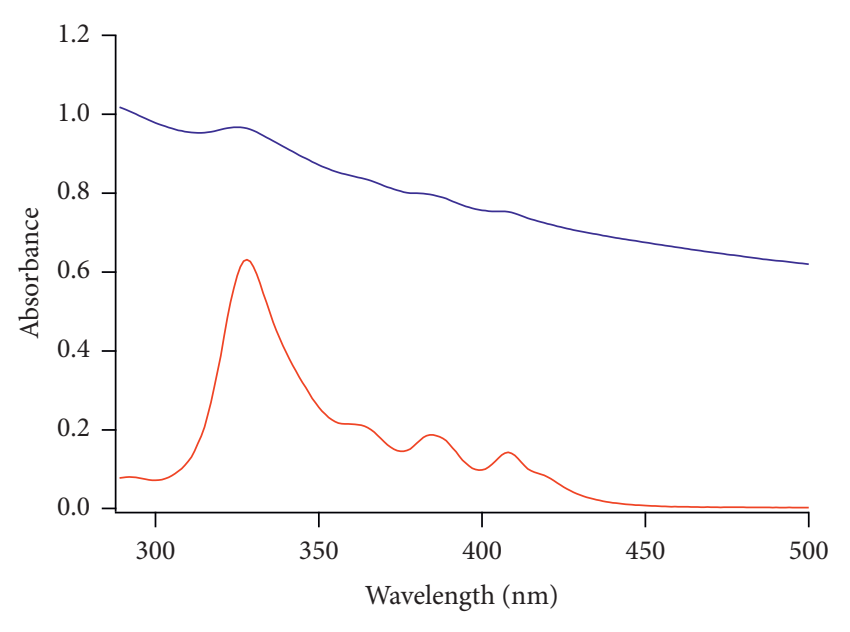

- AmB

— AmB-loaded MCPs

(a)

(b)

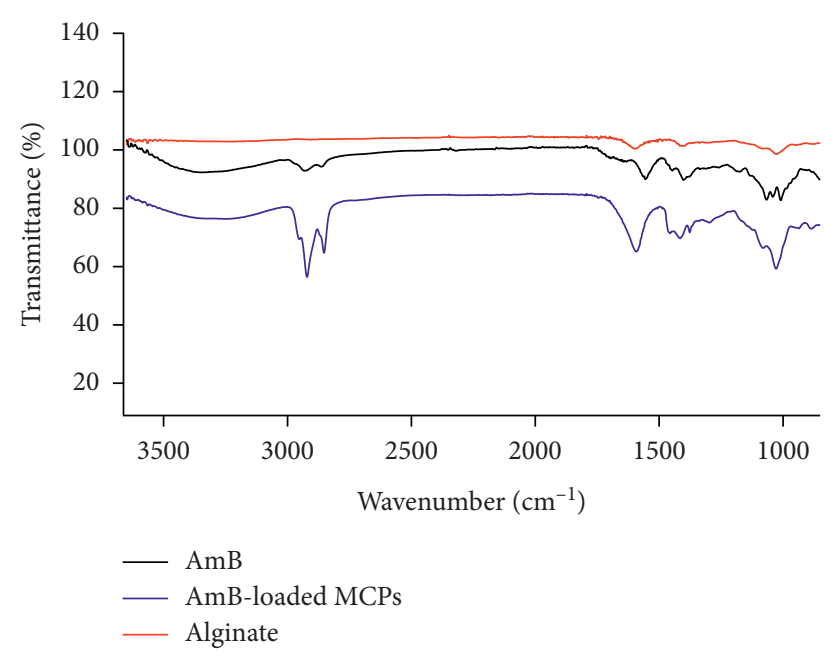

(c)

Figure 2: AmB-loaded MCPs characterization. (a) Hydrodynamic diameter of AmB-loaded MCPs. (b) Absorption spectra of MCPs-AmB and free AmB. (c) FTIR spectra of AmB-loaded MCPs, free AmB, and alginate.

used to evaluate the antifungal effectiveness of the microplatform developed in this work. Cells were exposed to two concentrations of $\mathrm{AmB}(0.5 \mu \mathrm{g} / \mathrm{mL}$ or $4 \mu \mathrm{g} / \mathrm{mL})$ for 48 hours at $35^{\circ} \mathrm{C}$ and the viability ratio and $\mathrm{CFU} / \mathrm{mL}$ were determined using the cell counting method and plate count method, respectively. These concentrations were chosen in accordance to clinical concentrations reported in the literature $[50,51]$. The cytotoxicity of blank MCPs at the corresponding concentration used for $0.5 \mu \mathrm{g} / \mathrm{mL}$ and $4 \mu \mathrm{g} /$ $\mathrm{mL}$ was also evaluated. The results demonstrated that the efficacy of AmB-loaded MCPs is similar to that of free AmB (Figure 5). No statistically significant difference in viability ratio or $\mathrm{CFU} / \mathrm{mL}$ was observed between cells treated with free AmB and cells treated with AmB-loaded MCPs at $0.5 \mu \mathrm{g} / \mathrm{mL}$ or $4 \mu \mathrm{g} / \mathrm{mL}$. MCPs did not induce any significant cytotoxicity on Candida albicans cells at the concentration used in this work. This result confirmed that the decrease on cell viability is exclusively related to the AmB released from the alginate microparticles. The effectiveness of the platform developed in this work using the emulsification/ external gelation method is higher when compared to other alginate-based platforms reported in the literature. For example, the in vitro antifungal activity of alginate-based nanoparticles containing miltefosine against Candida spp. was lower than that of the free drug [36]. A similar behavior was observed for $A m B$ conjugates that were obtained by reacting $A m B$ with sodium alginate via imine and amine linkages [42]. This high antifungal efficacy may be attributed to the fabrication procedure. It is known that the production methods of alginate nano- or microparticles have a significant impact on tuning the (bio)pharmaceutical performance [44]. Based on these results, this platform may not require repeated intravenous injections which is one of the limitations of commercial lipid formulations.

Additionally, several studies have reported that the utilization of carrier systems, such as liposomes, 


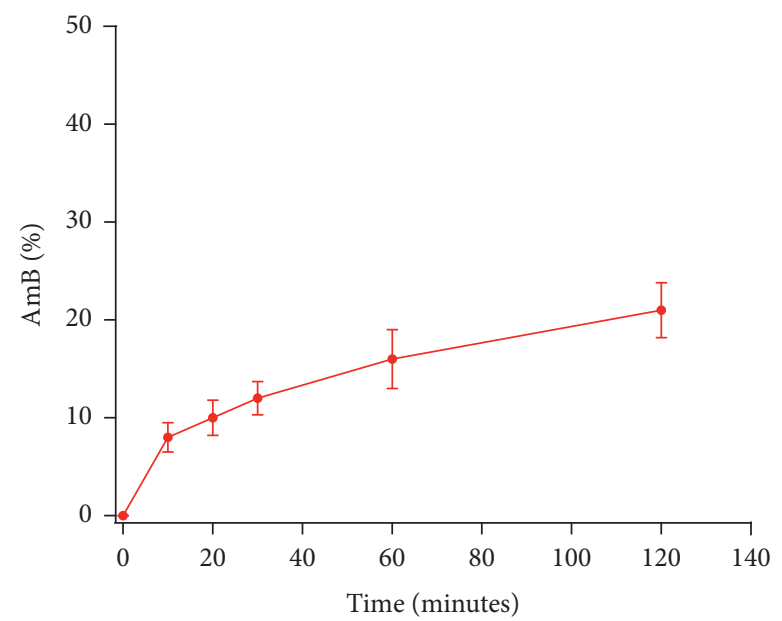

FIGURE 3: Release profile of AmB-loaded MCPs. Error bars represent standard deviation of three independent experiments.

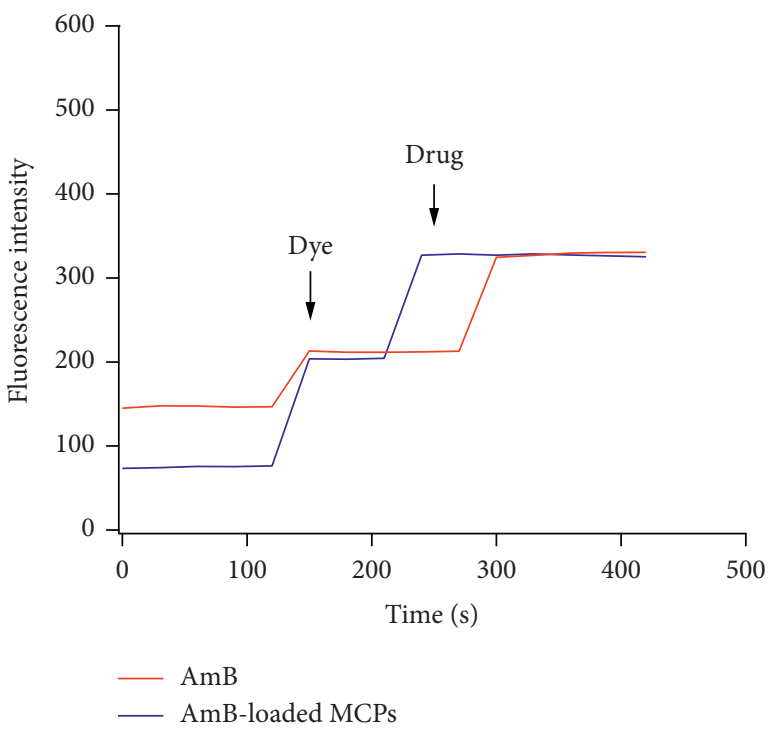

Figure 4: Membrane depolarization of Candida albicans cells by AmB-MCPs and free AmB using $\operatorname{DiBAC}_{4}(3)$ dye.

nanoparticles, and micelles, has reduced the main side effects of $\mathrm{AmB}[3,26,60,61]$. Thus, the use of the alginate-based drug delivery system could decrease significantly the side effects of this drug while inducing a therapeutic effect similar to the free AmB.

3.5. In Vitro Cytotoxicity of AmB-Loaded MCPs in Lung Cells. Nephrotoxicity has been the most studied side effect induced by polymeric-based drug delivery systems containing AmB. The majority of the studies related to the fabrication of these vehicles have focused on evaluating their in vitro toxicity in human kidney cells, demonstrating their ability to significantly decrease the main side effects of $A m B[25,26,42,52,53]$. Nevertheless, the toxicity caused by polymeric formulations in lung cells has not been a focus of study. It has been reported that although the encapsulation of $\mathrm{AmB}$ into drug delivery systems (lipid formulations) decreased the main side effects of this drug; these formulations induced acute pulmonary toxicity $[19,23,27-30]$. On the other hand, Sangeetha et al. reported that encapsulating AmB into alginate/poly-L-lysine nanoparticles impacted the biodistribution of the drug, inducing high accumulation of $\mathrm{AmB}$ in the lungs while reducing it in the kidneys [43]. Thus, it is necessary to study the in vitro toxicity of AmBloaded MCPs in lung cells which is the focus of our study. To this, human lung cells (H-460) were exposed to various concentrations of $\mathrm{AmB}(0-100 \mu \mathrm{g} / \mathrm{mL})$ for $48 \mathrm{~h}$. The amount of AmB-loaded MCPs and MCPs used corresponded to an equivalent amount of free $\mathrm{AmB}$ and the concentration used for AmB-loaded MCPs, respectively. After this period of time, the cell viability was determined using the CellTiter $96^{\circledR}$ AQueous assay. The results demonstrated that free AmB induced toxicity in a dosedependent manner with an IC50 of $50 \mu \mathrm{g} / \mathrm{mL}$ (Figure 6). This is consistent with previous reports which established that high concentrations of $\mathrm{AmB}$ induced lung cell injury $[29,62]$. On the other hand, blank microparticles (MCPs) and AmB-loaded MCPs did not induce lung cell toxicity in the range assessed. These results demonstrated that AmBloaded MCPs were able to induce antifungal toxicity against Candida albicans yeast cells at the same level of free $\mathrm{AmB}$ with minimal toxicity in lung cells, which has been one of the main concerns when $A m B$ is encapsulated into lipid-based drug delivery systems. Thus, this microplatform may not require several infusions for a successful treatment while reducing the pulmonary toxic effect induced by current commercial formulations.

3.6. Cell Membrane Injury. Although it is not clear why current AmB lipid formulations induce pulmonary toxicity, studies have reported that $\mathrm{AmB}$ directly injures cells in a dose- and time-dependent manner [29]. The injury could be caused by the formation of pores in cholesterol containing membranes [63]. Because it has been reported that alginate-based vehicles can accumulate at high 


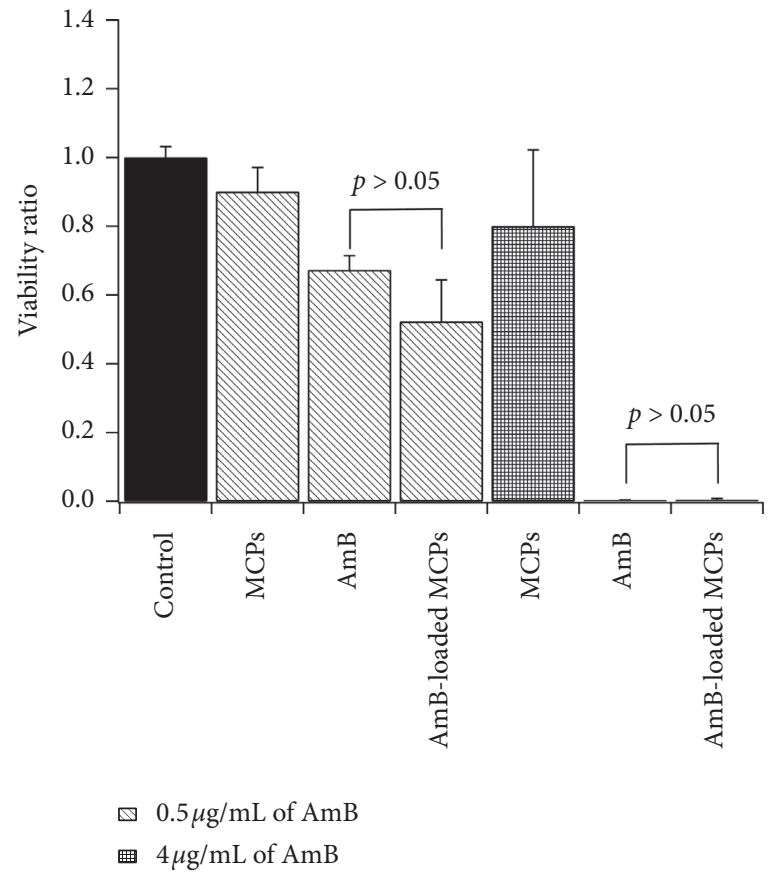

(a)

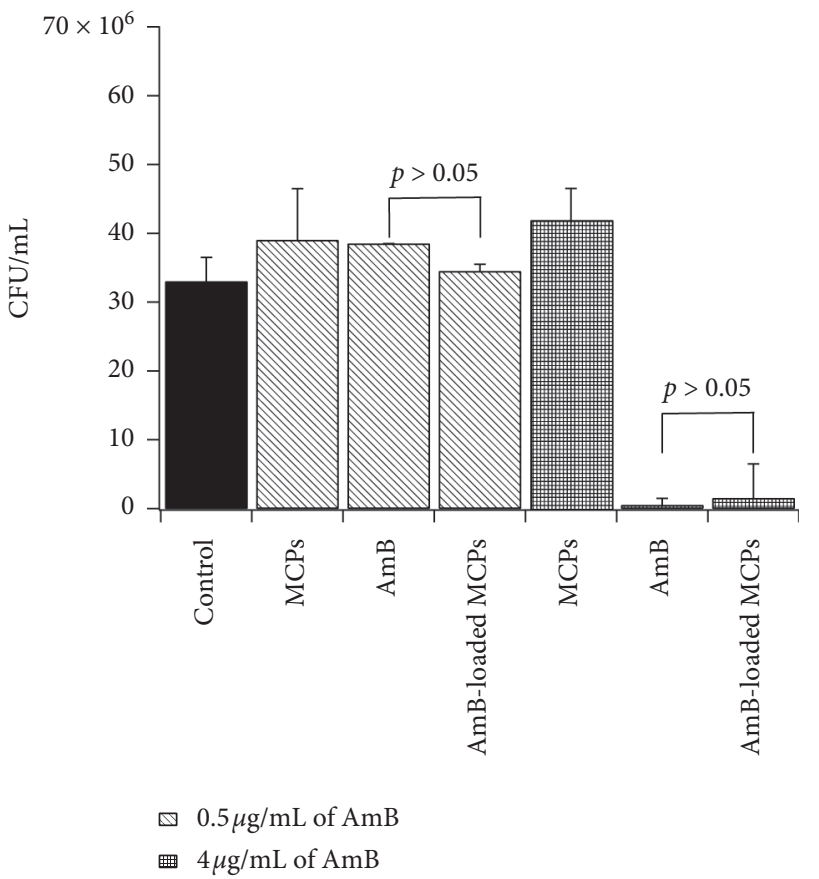

(b)

Figure 5: Cell viability: (a) viability ratio and (b) CFU/mL of Candida albicans cells treated with free AmB or AmB-loaded MCPs for 48 hours. The amount of AmB-loaded MCPs added corresponded to an equivalent amount of free AmB. MCPs represent blank microparticles at the concentration used for AmB-loaded MCPs. Error bars represent the standard error of two independent experiments.

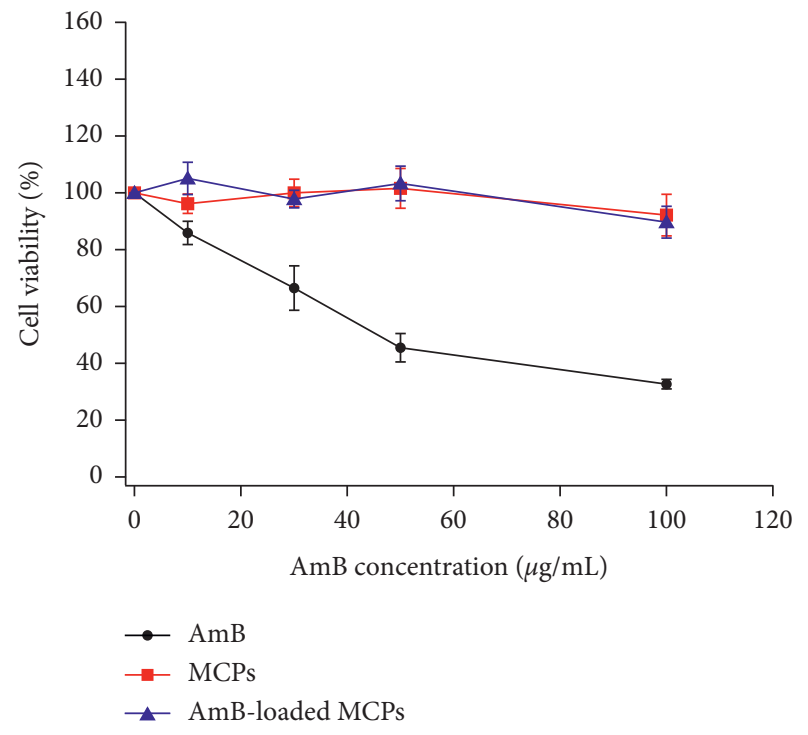

FIGURE 6: Cell viability of human lung cells treated with free AmB or AmB-loaded MCPs for 48 hours. The amount of AmB-loaded MCPs added corresponded to an equivalent amount of free AmB. MCPs represent blank microparticles at the concentration used for AmB-loaded MCPs. Error bars represent the standard error of four independent experiments.

concentrations in the lungs [43], qualitative diffusion analysis of acridine orange ( $\mathrm{AO}$ ) was performed in order to confirm that the vehicle developed in this work does not induce significant cell membrane damage. AO diffuses passively through the cellular membrane and it has been used to evaluate qualitatively membrane perturbation or damage [64]. If pores are formed on the cell membrane, the intracellular accumulation of $\mathrm{AO}$ will increase. Thus, an increase in the fluorescence intensity will be observed as it is directly proportional to the amount of dye that has entered into the cell. Our results demonstrated that, after exposing lung cells to $\mathrm{AmB}$ at $50 \mu \mathrm{g} / \mathrm{ml}$ for 24 hours, the free drug had the ability to significantly increase the incorporation of $\mathrm{AO}$ into the cells when compared to untreated cells (Figure 7). Cells treated with AmB-loaded MCPs showed a slight increase in AO fluorescence when compared to the control. These results demonstrated that the free drug was able to significantly enhance the rate of passive diffusion of $\mathrm{AO}$ across the membrane, presumably due to severe cell membrane damage. On the other hand, the cell membrane damage caused by the alginate-based vehicle was minimal. It has been reported that the severity of the cell membrane damage is related to cell survival [65]. Cells have the ability to repair the cell membrane when minor damage occurs; nevertheless, when the damage is beyond their repair capacity, cell death will be induced [65]. Therefore, the cell damage caused by the alginate vehicle seems to be minor allowing the cell to repair the membrane. This had a positive impact on the cell viability as demonstrated by the toxicity assay. Taken together, the toxicity assay results and cell membrane injury studies indicate that the encapsulation of $\mathrm{AmB}$ into alginate microparticles using the emulsification/external gelation method may induce minimal pulmonary toxicity. 

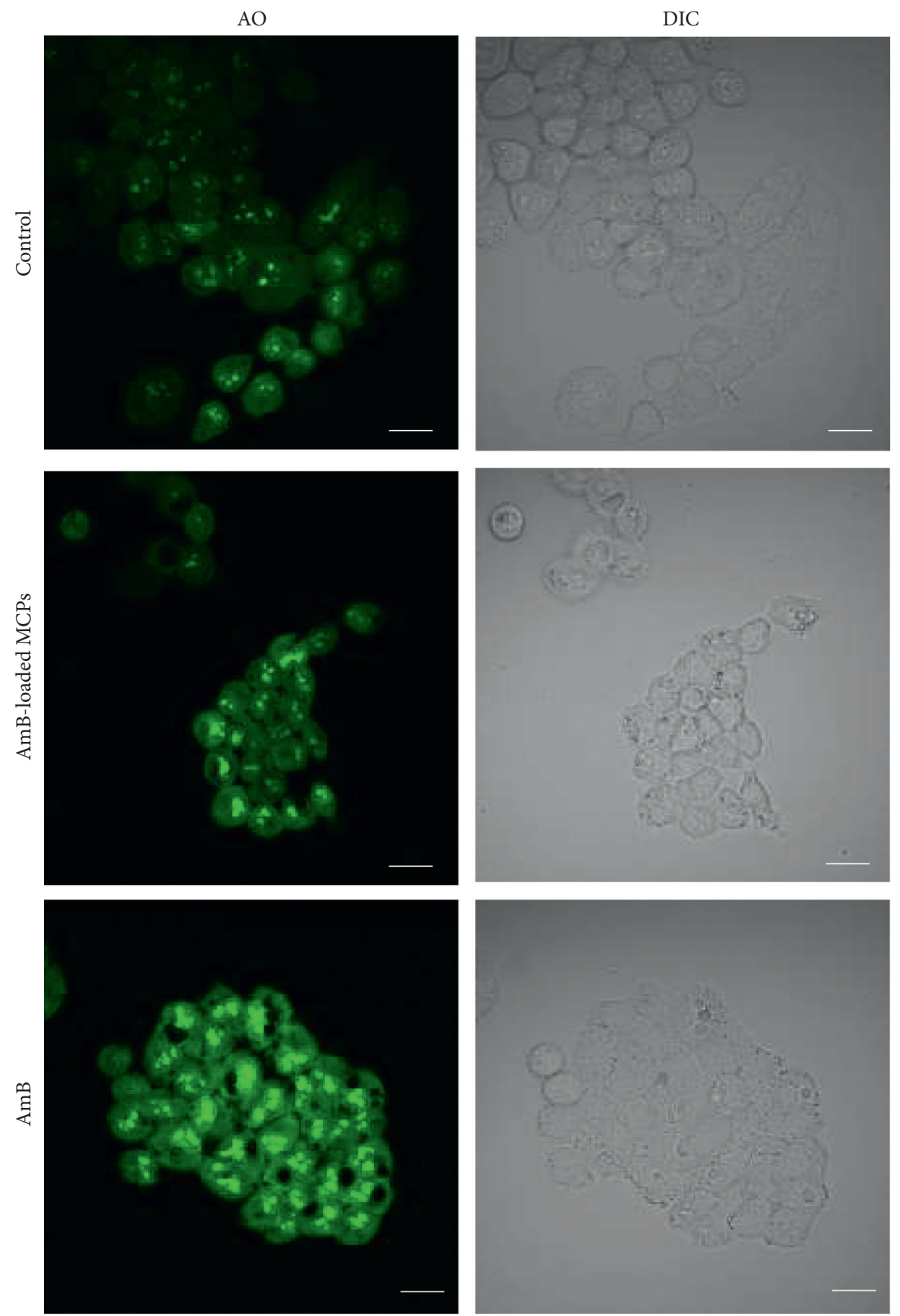

Figure 7: Acridine orange incorporation into lung cells treated with free AmB or AmB-loaded MCPs. Scale bar represents $20 \mu \mathrm{m}$.

\section{Conclusions}

Our work demonstrated that AmB-loaded MCPs were effective in disrupting the cellular membrane potential and reducing the cell viability of Candida albicans cells at the same level as free AmB with minimal lung cell toxicity and cell membrane injury. These results indicate that the activity of the antifungal was not compromised during the fabrication process and the microplatform may not require several infusions for a successful treatment while reducing the pulmonary toxic effect induced by current commercial lipid formulations.

Although the antifungal toxicity induced by the free drug and AmB-loaded MCPs is statistically the same, one of the main side effects induced by current AmB-lipid formulations (pulmonary toxicity) may be reduced by encapsulating $\mathrm{AmB}$ into alginate microparticles using the emulsification/ external gelation method.

Additionally, this microvehicle can be modified to provide targeting abilities to further impact the biodistribution of drugs and allow better selectivity. Future work will be focused on modifying the platform with targeting molecules to render target-specific properties toward Candida albicans cells and evaluate its effectiveness and impact on pulmonary toxicity. Overall, our results provide convincing evidence of the effectiveness of the platform developed in this work toward Candida albicans cells and its ability to likely reduce pulmonary toxicity. 


\section{Data Availability}

All data included in our manuscript are available.

\section{Conflicts of Interest}

The authors declare no conflicts of interest.

\section{Acknowledgments}

The authors thank the US National Science Foundation (instrument grant award 1827921) and Inter American University of Puerto Rico (Seed Grant Funding) for supporting this research work.

\section{References}

[1] Centers for Disease Control and Prevention, Fungal Diseases, Centers for Disease Control and Prevention, Atlanta, GA, USA, 2019.

[2] J. Tollemar, L. Klingspor, and O. Ringdén, "Liposomal amphotericin B (AmBisome) for fungal infections in immunocompromised adults and children," Clinical Microbiology and Infection, vol. 7, no. Suppl 2, pp. 68-79, 2001.

[3] M. Yang, "Synergistic antifungal effect of amphotericin B-loaded poly(lactic-Co-glycolic acid) nanoparticles and ultrasound against Candida albicans biofilms," Antimicrob Agents Chemother, vol. 63, no. 4, 2019.

[4] G. Quindós, "Epidemiology of candidaemia and invasive candidiasis. A changing face," Revista Iberoamericana de Micología, vol. 31, no. 1, pp. 42-48, 2014.

[5] C. R. Sims, L. Ostrosky-Zeichner, and J. H. Rex, "Invasive candidiasis in immunocompromised hospitalized patients," Archives of Medical Research, vol. 36, no. 6, pp. 660-671, 2005.

[6] S. Tsay, S. Williams, Y. Mu et al., "363. national burden of candidemia, United States, 2017," Open Forum Infectious Diseases, vol. 5, no. suppl_1, pp. S142-S143, 2018.

[7] E. Ricotta, Y. L. Lai, S. S. Kadri, M. Lionakis, D. R. Prevots, and J. Adjemian, "362. species distribution and trends of invasive candidiasis in the United States, 2009-2015, using a large electronic medical record database," Open Forum Infectious Diseases, vol. 5, no. suppl_1, p. S142, 2018.

[8] M. A. Pfaller and D. J. Diekema, "Epidemiology of invasive candidiasis: a persistent public health problem," Clinical Microbiology Reviews, vol. 20, no. 1, pp. 133-163, 2007.

[9] C. F. Rodrigues, M. E. Rodrigues, and M. Henriques, "Candida sp. infections in patients with diabetes mellitus," Journal of Clinical Medicine, vol. 8, no. 1, 2019.

[10] A. Halperin, Y. Shadkchan, E. Pisarevsky et al., "Novel watersoluble amphotericin B-PEG conjugates with low toxicity and potentin VivoEfficacy," Journal of Medicinal Chemistry, vol. 59, no. 3, pp. 1197-1206, 2016.

[11] C. Barau, J. Braun, C. Vincent et al., "Pharmacokinetic study of raltegravir in HIV-infected patients with end-stage liver disease: the LIVERAL-ANRS 148 study," Clinical Infectious Diseases, vol. 59, no. 8, pp. 1177-1184, 2014.

[12] M. Liu, M. Chen, and Z. Yang, "Design of amphotericin B oral formulation for antifungal therapy," Drug Delivery, vol. 24, no. 1, pp. 1-9, 2017.

[13] Z. Xiao, "Epidemiology, species distribution, antifungal susceptibility and mortality risk factors of candidemia among critically ill patients: a retrospective study from 2011 to 2017 in a teaching hospital in China," Antimicrob Resist Infect Control, vol. 8, p. 89, 2019.
[14] P. G. Pappas, "Invasive candidiasis," Nature Reviews Disease Primers, vol. 4, p. 18026, 2018.

[15] C.-C. Lai, C.-K. Tan, Y.-T. Huang, P.-L. Shao, and P.-R. Hsueh, "Current challenges in the management of invasive fungal infections," Journal of Infection and Chemotherapy, vol. 14, no. 2, pp. 77-85, 2008.

[16] J. E. Berdal, "Nosocomial candidemia; risk factors and prognosis revisited; 11 years experience from a Norwegian secondary hospital," PLoS One, vol. 9, no. 7, Article ID e103916, 2014.

[17] S. S. Magill, E. O'Leary, S. J. Janelle et al., "Changes in prevalence of health care-associated infections in U.S. Hospitals," New England Journal of Medicine, vol. 379, no. 18, pp. 1732-1744, 2018.

[18] B. Chudzik, "Synergistic antifungal interactions of amphotericin B with 4-(5-methyl-1,3,4-thiadiazole-2-yl) benzene1,3-diol," Scientific Reports, vol. 9, no. 1, p. 12945, 2019.

[19] S. Keane, P. Geoghegan, P. Povoa, S. Nseir, A. Rodriguez, and I. Martin-Loeches, "Systematic review on the first line treatment of amphotericin B in critically ill adults with candidemia or invasive candidiasis," Expert Review of Antiinfective Therapy, vol. 16, no. 11, pp. 839-847, 2018.

[20] G. Cuddihy, "The development of oral amphotericin B to treat systemic fungal and parasitic infections: has the myth been finally realized?," Pharmaceutics, vol. 11, no. 3, 2019.

[21] A. Noor and C. V. Preuss, "Antifungal Membrane Function Inhibitors (Amphotericin B)," StatPearls Publishing LLC., Treasure Island, FL, USA, 2019.

[22] R. Laniado-Laborín and M. N. Cabrales-Vargas, "Amphotericin B: side effects and toxicity," Revista Iberoamericana de Micología, vol. 26, no. 4, pp. 223-227, 2009.

[23] G. P. Patel, C. W. Crank, and J. B. Leikin, "An evaluation of hepatotoxicity and nephrotoxicity of liposomal amphotericin B (L-AMB)," Journal of Medical Toxicology, vol. 7, no. 1, pp. 12-15, 2011.

[24] F. Saliba and B. Dupont, "Renal impairment and amphotericin B formulations in patients with invasive fungal infections," Medical Mycology, vol. 46, no. 2, pp. 97-112, 2008.

[25] Q. Zia, "Biomimetically engineered amphotericin B nanoaggregates circumvent toxicity constraints and treat systemic fungal infection in experimental animals," Scientific Reports, vol. 7, no. 1, p. 11873, 2017.

[26] L. Zhou, P. Zhang, Z. Chen et al., "Preparation, characterization, and evaluation of amphotericin B-loaded MPEGPCL-g-PEI micelles for local treatment of oral Candida albicans," International Journal of Nanomedicine, vol. 12, pp. 4269-4283, 2017.

[27] S. J. Levine, "Cardiopulmonary toxicity after liposomal amphotericin B infusion," Annals of Internal Medicine, vol. 114, no. 8, pp. 664-666, 1991.

[28] R. H. Haber, "Acute pulmonary decompensation due to amphotericin B in the absence of granulocyte transfusions," The New England Journal of Medicine, vol. 315, no. 13, p. 836, 1986.

[29] J. Collazos, E. Martínez, J. Mayo, and S. Ibarra, "Pulmonary reactions during treatment with amphotericin B: review of published cases and guidelines for management," Clinical Infectious Diseases, vol. 33, no. 7, pp. E75-e82, 2001.

[30] M. Arning, A. H. Heer-Sonderhoff, A. Wehmeier, and W. Schneider, "Pulmonary toxicity during infusion of liposomal amphotericin B in two patients with acute leukemia," European Journal of Clinical Microbiology \& Infectious Diseases, vol. 14, no. 1, pp. 41-43, 1995.

[31] P. Schneider, R. M. Klein, L. Dietze, D. Söhngen, M. Leschke, and A. Heyll, "Anaphylactic reaction to liposomal 
amphotericin (AmBisome)," British Journal of Haematology, vol. 102, no. 4, pp. 1108-1109, 1998.

[32] R. B. S. Laing, L. J. R. Milne, C. L. S. Leen, G. P. Malcolm, and A. J. W. Steers, "Anaphylactic reactions to liposomal amphotericin," The Lancet, vol. 344, no. 8923, p. 682, 1994.

[33] J. Shuster, "Amphotericin B lipid complex and respiratory distress," Nursing, vol. 29, no. 3, p. 70, 1999.

[34] C. C. Spadari, L. B. Lopes, and K. Ishida, "Potential use of alginate-based carriers as antifungal delivery system," Frontiers in Microbiology, vol. 8, p. 97, 2017.

[35] A. A. Khan, M. Jabeen, A. M. Alanazi, and A. A. Khan, "Antifungal efficacy of amphotericin B encapsulated fibrin microsphere for treating Cryptococcus neoformans infection in Swiss albino mice," The Brazilian Journal of Infectious Diseases, vol. 20, no. 4, pp. 342-348, 2016.

[36] C. d. C. Spadari, F. W. M. d. S. de Bastiani, L. B. Lopes, and K. Ishida, "Alginate nanoparticles as non-toxic delivery system for miltefosine in the treatment of candidiasis and cryptococcosis," International Journal of Nanomedicine, vol. 14, pp. 5187-5199, 2019.

[37] K. Y. Lee and D. J. Mooney, "Alginate: properties and biomedical applications," Progress in Polymer Science, vol. 37, no. 1, pp. 106-126, 2012.

[38] P. Severino, C. F. da Silva, L. N. Andrade, D. de Lima Oliveira, J. Campos, and E. B. Souto, "Alginate nanoparticles for drug delivery and targeting," Current Pharmaceutical Design, vol. 25, no. 11, pp. 1312-1334, 2019.

[39] C. P. Reis, L. V. Roque, M. Baptista, and P. Rijo, "Innovative formulation of nystatin particulate systems in toothpaste for candidiasis treatment," Pharmaceutical Development and Technology, vol. 21, no. 3, pp. 282-287, 2016.

[40] S. K. Patel, D. R. Shah, and S. Tiwari, "Bioadhesive films containing fluconazole for mucocutaneous candidiasis," Indian Journal of Pharmaceutical sciences, vol. 77, no. 1, pp. 55-61, 2015.

[41] Z. Ahmad, S. Sharma, and G. K. Khuller, "Chemotherapeutic evaluation of alginate nanoparticle-encapsulated azole antifungal and antitubercular drugs against murine tuberculosis," Nanomedicine: Nanotechnology, Biology and Medicine, vol. 3, no. 3, pp. 239-243, 2007.

[42] V. Ravichandran and A. Jayakrishnan, "Synthesis and evaluation of anti-fungal activities of sodium alginate-amphotericin B conjugates," International Journal of Biological Macromolecules, vol. 108, pp. 1101-1109, 2018.

[43] S. Sangeetha, D. N. Venkatesh, R. Adhiyaman, K. Santhi, and B. Suresh, "Formulation of sodium alginate nanospheres containing amphotericin B for the treatment of systemic candidiasis," Tropical Journal of Pharmaceutical Research, vol. 6, pp. 653-659, 2007.

[44] A. Sosnik, "Alginate particles as platform for drug delivery by the oral route: state-of-the-art," ISRN Pharmaceutics, vol. 2014, Article ID 926157, 17 pages, 2014.

[45] G. Fundueanu, C. Nastruzzi, A. Carpov, J. Desbrieres, and M. Rinaudo, "Physico-chemical characterization of Ca-alginate microparticles produced with different methods," Biomaterials, vol. 20, no. 15, pp. 1427-1435, 1999.

[46] S. Bhatia, "Significance of algal polymer in designing amphotericin B nanoparticles," The Scientific World Journal, vol. 2014, Article ID 564573, 21 pages, 2014.

[47] G. Vandermeulen, "Encapsulation of amphotericin B in poly(ethylene glycol)-block-poly(epsilon-caprolactone-cotrimethylenecarbonate) polymeric micelles," International Journal of Pharmaceutics, vol. 309, no. 1-2, pp. 234-240, 2006.
[48] J. Cho, H. Choi, J. Lee, M.-S. Kim, H.-Y. Sohn, and D. G. Lee, "The antifungal activity and membrane-disruptive action of dioscin extracted from Dioscorea nipponica," Biochimica et Biophysica Acta (BBA)-Biomembranes, vol. 1828, no. 3, pp. 1153-1158, 2013.

[49] D. S. Adams and M. Levin, "Measuring resting membrane potential using the fluorescent voltage reporters DiBAC4(3) and CC2-DMPE," Cold Spring Harbor Protocols, vol. 2012, no. 4, pp. 459-464, 2012.

[50] S. Harmsen, A. C. McLaren, C. Pauken, and R. McLemore, "Amphotericin B is cytotoxic at locally delivered concentrations," Clinical Orthopaedics and Related Research, vol. 469, no. 11, pp. 3016-3021, 2011.

[51] V. Al Jalali, R. Sauermann, S. Eberl, and M. Zeitlinger, "In vitro activity of voriconazole and amphotericin B against Candida albicans, Candida krusei, and Cryptococcus neoformans in human cerebrospinal fluid," Infection, vol. 47, no. 4, pp. 565-570, 2019.

[52] S. Kagan, D. Ickowicz, M. Shmuel et al., "Toxicity mechanisms of amphotericin B and its neutralization by conjugation with arabinogalactan," Antimicrobial Agents and Chemotherapy, vol. 56, no. 11, pp. 5603-5611, 2012.

[53] T. R. Tan, "Characterization of a polyethylene glycolamphotericin B conjugate loaded with free AMB for improved antifungal efficacy," PLoS One, vol. 11, no. 3, Article ID e0152112, 2016.

[54] P. S. Hiemstra, G. Grootaers, A. M. van der Does, C. A. M. Krul, and I. M. Kooter, "Human lung epithelial cell cultures for analysis of inhaled toxicants: lessons learned and future directions," Toxicology in Vitro, vol. 47, pp. 137-146, 2018.

[55] E. Joseph and S. Gautam, "Multifunctional nanocrystals for cancer therapy: a potential nanocarrier," in Nanomaterials for Drug Delivery and Therapy, Elsevier, Amsterdam, Netherlands, pp. 91-116, 2019.

[56] A. Kumar and K. D. Chandra, "Methods for characterization of nanoparticles," in Advances in Nanomedicine for the Delivery of Therapeutic Nucleic Acids, Woodhead Publishing, Cambridge, UK, pp. 43-58, 2017.

[57] M. Gagos, "Binding of antibiotic amphotericin B to lipid membranes: monomolecular layer technique and linear dichroism-FTIR studies," Molecular Membrane Biology, vol. 22, no. 5, pp. 433-442, 2005.

[58] K. Rima, M. Dima, and Y. Paolo, "Polycaprolactone as drug carrier for an antifungal agent," Journal of Drug Delivery and Therapeutics, vol. 8, no. 1, pp. 81-85, 2018.

[59] R. S. Liao, R. P. Rennie, and J. A. Talbot, "Assessment of the effect of amphotericin B on the vitality of Candida albicans," Antimicrobial Agents and Chemotherapy, vol. 43, no. 5, pp. 1034-1041, 1999.

[60] J. Roberts, J. Bingham, A. C. McLaren, and R. McLemore, "Liposomal formulation decreases toxicity of amphotericin B in vitro and in vivo," Clinical Orthopaedics and Related Research, vol. 473, no. 7, pp. 2262-2269, 2015.

[61] C. Alvarez, D. H. Shin, and G. S. Kwon, "Reformulation of fungizone by PEG-DSPE micelles: deaggregation and detoxification of amphotericin B," Pharmaceutical Research, vol. 33, no. 9, pp. 2098-2106, 2016.

[62] M. Cutaia, S. R. Bullard, K. Rudio, and S. Rounds, "Characteristics of amphotericin B-induced endothelial cell injury," The Journal of Laboratory and Clinical Medicine, vol. 121, no. 2, pp. 244-256, 1993.

[63] B. Venegas, J. González-Damián, H. Celis, and I. OrtegaBlake, "Amphotericin B channels in the bacterial membrane: 
role of sterol and temperature," Biophysical Journal, vol. 85, no. 4, pp. 2323-2332, 2003.

[64] M. P. Alvarez-Berrios, "Hyperthermic potentiation of cisplatin by magnetic nanoparticle heaters is correlated with an increase in cell membrane fluidity," International Journal of Nanomedicine, vol. 8, pp. 1003-1013, 2013.

[65] A. Etxaniz, "Membrane repair mechanisms against permeabilization by pore-forming toxins," Toxins (Basel), vol. 10, no. 6,2018 . 